\title{
Fractional-Order Adaptive Fault Estimation for a Class of Nonlinear Fractional-Order Systems
}

\author{
Ibrahima N'Doye and Taous-Meriem Laleg-Kirati
}

\begin{abstract}
This paper studies the problem of fractional-order adaptive fault estimation for a class of fractional-order Lipschitz nonlinear systems using fractional-order adaptive fault observer. Sufficient conditions for the asymptotical convergence of the fractional-order state estimation error, the conventional integer-order and the fractional-order faults estimation error are derived in terms of linear matrix inequalities (LMIs) formulation by introducing a continuous frequency distributed equivalent model and using an indirect Lyapunov approach where the fractional-order $\alpha$ belongs to $0<\alpha<1$. A numerical example is given to demonstrate the validity of the proposed approach.
\end{abstract}

Index Terms-Fractional-order nonlinear system, fractionalorder adaptive fault estimation, actuator fault, observer design, indirect Lyapunov approach, linear matrix inequality (LMI).

\section{INTRODUCTION}

Over the past couple of decades, fractional calculus has gained significant attention as one of the topics that can be applied to a variety of fields in engineering. Many systems can be described with the help of fractional derivatives. These systems are known to display fractional-order dynamics : electromagnetic systems [1], dielectric polarization [2], viscoelastic systems [3]. In biology, it has been shown that the cell membranes of biological organisms have a fractionalorder electrical conductance [4]. In economics, it is known that some financial systems can display fractional order dynamics [5]. The advantage of using fractional differential equations in these and other applications is their non-local property, fractional-order derivatives provide a more appropriate tool for modeling different phenomena.

Using the frequency distributed fractional integrator equivalent model, an indirect Lyapunov method to fractional nonlinear differential equations was addressed in [6]. The design of state estimators is one of the essential points in control theory whose solution, in the linear case, is the well known Luenberger observer. Many contributions to the extension of the Luenberger observer for nonlinear systems have been proposed in the literature (see [7], [8], [9], [10] and references therein). The observer-based control is usually applied when we do not have access to all the states of a system. The notion of detectability which has been extended to nonlinear systems in [11], [12] allows to design nonlinear observer-based controls using the separation principle.

Ibrahima N'Doye and Taous-Meriem Laleg-Kirati are with Computer, Electrical and Mathematical Sciences and Engineering Division (CEMSE), King Abdullah University of Science and Technology(KAUST). ibrahima.ndoye@kaust.edu.sa taousmeriem.laleg@kaust.edu.sa
Recently, there were much research on observer design for nonlinear systems in both integral-order and fractional-order cases. Using indirect Lyapunov method and LMI techniques, Boroujeni et al. [13] and Lan et al. [14] investigated the observer design for fractional-order nonlinear systems.

The advantage of an adaptive fault diagnosis observer is that the state vector estimation and actuator fault estimation can be obtained simultaneously [15]. The main problems in the use of the conventional adaptive observer-based fault estimation are first to achieve the performance requirements of fault estimation, i.e, rapidity and accuracy, and second to fulfill the stringent equation and solve them to obtain the design parameters. Recently, adaptive parameter estimation problems for some classes of linear fractional-order processes have been discussed in [16]. In [17], the design of a fractional-order differentiator for a class of signals satisfying a linear differential equation with unknown parameters has been proposed using a set of modulating functions.

The aim of this paper is to develop a strategy for conventional integer-order and fast fractional-order faults estimation of fractional-order nonlinear systems locally involving Lipschitz by using an indirect Lyapunov approach. To our best knowledge, the fractional-order adaptive fault estimation for a class of fractional-order Lipschitz nonlinear systems using adaptive fractional-order fault observer is still open problem.

This paper is organized as follows. In section II, some basic definitions and properties on the fractional integral and derivative, and problem formulation are presented. In section III, the main results are derived, and then sufficient conditions for the convergence of the conventional integer-order and the fractional-order faults estimation error dynamics are derived in terms of linear matrix inequalities (LMIs). In section IV, an example is presented to illustrate our proposed results. Finally, some conclusions and future perspectives are drawn in section $\mathrm{V}$.

Notations. $M^{T}$ is the transpose of $M, *$ denotes convolution operator and $D^{\alpha}$ represents initialized $\alpha^{\text {th }}$ order differintegration. In symmetric block matrices, the operator $\bullet$ represents a term induced by symmetry.

\section{BASIC DEFINITION AND PRELIMINARY RESULTS}

In this section, we present some basic definitions and properties on the fractional integration and derivation.

\section{A. Fractional integration and derivation}

Fractional-order integration and differentiation are the generalization of their integer-order counterparts. Efforts to 
extend the specific definitions of the traditional integer order to the more general arbitrary order context led to different definitions for fractional derivatives [18]. One of the basic functions of fractional calculus is Euler's Gamma function which is defined by the integral

$$
\Gamma(z)=\int_{0}^{\infty} e^{-t} t^{z-1} \mathrm{~d} t
$$

which converges in the right half of the complex plane, i.e $\operatorname{Re}(z)>0$.

Definition 1: [19] The definition of fractional integral of continuous function $f(t)$ with respect to $t$ and the terminal value $t_{0}$ is given by

${ }_{t_{0}} D_{t}^{-\alpha} f(t)=\frac{1}{\Gamma(\alpha)} \int_{t_{0}}^{t}(t-\tau)^{\alpha-1} f(\tau) \mathrm{d} \tau, \quad \alpha>0$,

where $\Gamma($.$) is the well-known Gamma function which is$ defined in (1).

Definition 2: [19] The Riemann-Liouville derivative is defined by

$$
{ }_{t_{0}}^{R L} D_{t}^{\alpha} f(t)=D_{t_{0}}^{n} D_{t}^{\alpha-n} f(t), \quad n-1<\alpha<n,
$$

where $n \in \mathbb{Z}^{+}$and $D^{n}$ is the classical $n$-th order derivative.

Definition 3: [6] Let $h(t)$ be the impulse response of a linear system. The diffusive representation (or frequency weighting function) of $h(t)$ is called $\mu(\omega)$ with the following relation

$$
h(t)=\int_{0}^{\infty} \mu(\omega) e^{\omega t} \mathrm{~d} \omega
$$

where $\omega$ is the elementary frequency.

Remark 1: [6] The fractional order integral operator ${ }_{t_{0}} D_{t}^{-\alpha} f(t)$ can be written as

$$
{ }_{t_{0}} D_{t}^{-\alpha} f(t)=h(t) * f(t),
$$

where $*$ denotes the convolution operator and $h(t)=\frac{t^{\alpha-1}}{\Gamma(\alpha)}$, while the diffusive representation of $h(t)$ is defined as

$$
\mu(\omega)=\frac{\sin (\alpha \pi)}{\pi} \omega^{-\alpha}
$$

Lemma 1: [6] The fractional-order nonlinear differential equation

$$
{ }_{t_{0}} D_{t}^{\alpha} x(t)=f(x(t)),
$$

due to the continuous frequency distributed model of the fractional integrator, can be written as

$$
\left\{\begin{array}{l}
\frac{\partial z(\omega, t)}{\partial t}=-\omega z(\omega, t)+f(x(t)) \\
x(t)=\int_{0}^{\infty} \mu(\omega) z(\omega, t) \mathrm{d} \omega
\end{array}\right.
$$

where $z(\omega, t)$ is the infinite dimension distributed state variable and $\mu(\omega)$ is defined in (6).

In the following, $D^{\alpha}$ is used to denote the Rieman-Liouville fractional derivative of order $\alpha$.

The following lemma proved in [20] will be used in the next section.

Lemma 2: [20] For any matrices $X$ and $Y$ with appropriate dimensions, we have

$$
X^{T} Y+Y^{T} X \leqslant \delta X^{T} X+\delta^{-1} Y^{T} Y
$$

for any $\delta>0$.

Consider the following fractional-order systems with actuator fault

$$
\left\{\begin{array}{l}
D^{\alpha} x(t)=A x(t)+\Phi(x(t), u(t))+G f(t) \\
y(t)=C x(t)
\end{array} \quad 0<\alpha<1\right.
$$

where $x(t) \in \mathbb{R}^{n}$ is the state vector, $u(t) \in \mathbb{R}^{m}$ is the control input and $y(t) \in \mathbb{R}^{p}$ is the measurable output. $A \in$ $\mathbb{R}^{n \times n}, C \in \mathbb{R}^{m \times n}$ and $G \in \mathbb{R}^{n \times r}$ are constant matrices. $f(t) \in \mathbb{R}^{r}$ is the process or actuator fault and $\left\|D^{\alpha} f(t)\right\| \leqslant$ $f_{0}$. The number of output channels is greater than or equal to the number of fault inputs, i.e $p \geqslant r . \Phi(x(t), u(t))$ is a continuous nonlinear vector function, assumed to be Lipschitz, with a Lipschitz constant $\lambda$, i.e

$$
\left\|\Phi\left(x_{1}, u^{*}\right)-\Phi\left(x_{2}, u^{*}\right)\right\| \leqslant \lambda\left\|x_{1}-x_{2}\right\| .
$$

Here, it is assumed that the matrix pair $(C, A)$ is observable and the matrices $C$ and $G$ are of full rank, i.e $\operatorname{rank} C=p$ and $\operatorname{rank} G=r$. The necessary and sufficient conditions for stability of fractional-order observer can be formulated for the above conditions [21], [22]

i) $\operatorname{rank}(C G)=\operatorname{rank}(G)=r$,

ii) The triplet $(A, G, C)$ is minimum phase i.e.

$$
\begin{aligned}
\operatorname{rank}\left[\begin{array}{cc}
\sigma I_{n}-A & G \\
C & 0
\end{array}\right]= & n+\operatorname{rank}(G), \\
& \forall \sigma \in \mathbb{C},|\arg (\sigma)| \leqslant \alpha \frac{\pi}{2} .
\end{aligned}
$$

\section{MAIN RESULTS}

In this section, sufficient conditions for the convergence of the conventional integer-order and the fractional-order faults estimation error dynamics for nonlinear fractional-order systems are derived in terms of linear matrix inequalities (LMIs) formulation. In order to estimate the fault, we construct the following fractional-order fault estimation observer for system (10)

$$
\left\{\begin{array}{l}
D^{\alpha} \widehat{x}(t)=A \widehat{x}(t)+\Phi(\widehat{x}(t), u(t))+G \widehat{f}(t)-L(\widehat{y}(t)-y(t)) \\
\widehat{y}(t)=C \widehat{x}(t)
\end{array}\right.
$$

where $0<\alpha<1, \widehat{x}(t) \in \mathbb{R}^{n}$ is the observer state, $\widehat{y}(t) \in$ $\mathbb{R}^{p}$ is the observer output and $\widehat{f}(t) \in \mathbb{R}^{r}$ is an estimate of $f(t)$, and $L \in \mathbb{R}^{n \times p}$ is the gain matrix to be designed.

Let

$$
e_{x}(t)=\widehat{x}(t)-x(t), e_{y}(t)=\widehat{y}(t)-y(t), e_{f}(t)=\widehat{f}(t)-f(t)
$$

then the fractional-order error dynamics is given by

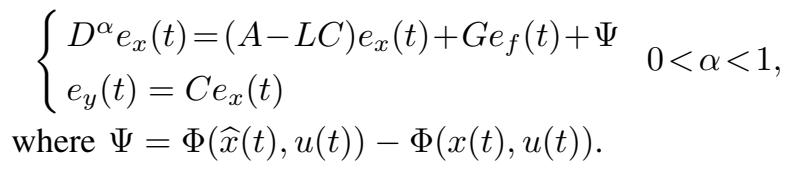

\section{A. Fractional-order adaptive fault estimation}

Generally speaking, constant fault, i.e $\dot{f}(t)=0$ is only considered based on the conventional integer-order algorithm, the derivative of $e_{f}(t)$ with respect to time is

$$
\dot{e}_{f}(t)=\dot{\widehat{f}}(t) \text {. }
$$


The asymptotical convergence of the fractional-order state estimation error and the fault estimation error are guaranteed by the following theorem.

Theorem 1: If there exists matrices $P=P^{T}>0 \in$ $\mathbb{R}^{n \times n}, X \in \mathbb{R}^{m \times n}, F \in \mathbb{R}^{r \times p}$ and a real scalar $\delta_{1}>0$ such that the following conditions hold

$$
\begin{gathered}
{\left[\begin{array}{cc}
P A+A^{T} P-X C-C^{T} X^{T}+\delta_{1} \lambda^{2} I & P \\
\bullet & -\delta_{1} I
\end{array}\right]<0,} \\
G^{T} P=F C .
\end{gathered}
$$

Then the adaptive fault estimation algorithm

$$
\dot{\hat{f}}(t)=-\Sigma F e_{y}(t),
$$

results in the state estimation error $e_{x}(t)$ and the fault estimation error $e_{f}(t)$ are asymptotically convergent where $\Sigma=\Sigma^{T}>0 \in \mathbb{R}^{r \times r}$ is the learning rate.

Moreover, the asymptotically stabilizing state-feedback gain matrix $L$ is given by

$$
L=P^{-1} X \text {. }
$$

Proof: It follows from lemma 1 that the fractional-order observer error dynamic system (14) can be written as

$$
\left\{\begin{array}{l}
\frac{\partial Z_{1}(\omega, t)}{\partial t}=-\omega Z_{1}(\omega, t)+\widetilde{A} e_{x}(t)+G e_{f}(t)+\Psi, \\
e_{x}(t)=\int_{0}^{\infty} \mu(\omega) Z_{1}(\omega, t) \mathrm{d} \omega
\end{array}\right.
$$

where $\widetilde{A}=A-L C$ and the fault estimation error (17) is given by

$$
e_{f}(t)=\widehat{f}(t)-f(t) .
$$

Let us define two Lyapunov functions

- $v(\omega, t)$ is the monochromatic Lyapunov function corresponding to the elementary frequency $\omega$.

- $V_{1}(t)$ is the Lyapunov function summing all the monochromatic $v(\omega, t)$ with the weighting function $\mu(\omega)$.

Thus, we can define our Lyapunov function as

$$
V(t)=\int_{0}^{\infty} \mu(\omega) Z_{1}^{T}(\omega, t) P Z_{1}(\omega, t) \mathrm{d} \omega+e_{f}^{T}(t) \Sigma^{-1} e_{f}(t) .
$$

The time derivative of $V(t)$ taken along the solution trajectories of (19) is

$$
\begin{aligned}
& \dot{V}(t)= \\
& \int_{0}^{\infty} \mu(\omega)\left(-\omega Z_{1}^{T}(\omega, t)+e_{x}^{T}(t) \widetilde{A}^{T}+e_{f}^{T}(t) G^{T}+\Psi^{T}\right) P Z_{1}(\omega, t) \mathrm{d} \omega \\
& +\int_{0}^{\infty} \mu(\omega) Z_{1}^{T}(\omega, t) P\left(-\omega Z_{1}(\omega, t)+\widetilde{A} e_{x}(t)+G e_{f}(t)+\Psi\right) \mathrm{d} \omega \\
& \quad-2 e_{f}^{T}(t) F C e_{x}(t) .
\end{aligned}
$$

Equation (22) can be written as

$$
\begin{aligned}
& \dot{V}(t)=-2 \int_{0}^{\infty} \omega \mu(\omega) Z_{1}^{T}(\omega, t) P Z_{1}(\omega, t) \mathrm{d} \omega \\
& +\int_{0}^{\infty} \mu(\omega) e_{x}^{T}(t) \widetilde{A}^{T} P Z_{1}(\omega, t) \mathrm{d} \omega+\int_{0}^{\infty} \mu(\omega) Z_{1}^{T}(\omega, t) P \widetilde{A} e_{x}(t) \mathrm{d} \omega \\
& +\int_{0}^{\infty} \mu(\omega) e_{f}^{T}(t) G^{T} P Z_{1}(\omega, t) \mathrm{d} \omega+\int_{0}^{\infty} \mu(\omega) Z_{1}^{T}(\omega, t) P G e_{f}(t) \mathrm{d} \omega \\
& +\int_{0}^{\infty} \mu(\omega) \Psi^{T} P Z_{1}(\omega, t) \mathrm{d} \omega+\int_{0}^{\infty} \mu(\omega) Z_{1}^{T}(\omega, t) P \Psi \mathrm{d} \omega
\end{aligned}
$$

$$
\begin{aligned}
& -2 e_{f}^{T}(t) F C e_{x}(t) . \\
= & -2 \int_{0}^{\infty} \mu(\omega) Z_{1}^{T}(\omega, t) P Z_{1}(\omega, t) \mathrm{d} \omega+e_{x}^{T}(t)\left(\widetilde{A}^{T} P+P \widetilde{A}\right) e_{x}(t) \\
& +2 e_{x}^{T}(t) P G e_{f}(t)-2 e_{f}^{T}(t) F C e_{x}(t)+\Psi^{T} P e_{x}(t)+e_{x}^{T}(t) P \Psi .
\end{aligned}
$$

Using (16b), it is easy to show that

$$
2 e_{x}^{T}(t) P G e_{f}(t)=2 e_{f}^{T}(t) F C e_{x}(t) .
$$

Then from (23), one can further obtain

$$
\begin{aligned}
& \dot{V}(t)=-2 \int_{0}^{\infty} \omega \mu(\omega) Z_{1}^{T}(\omega, t) P Z_{1}(\omega, t) \mathrm{d} \omega \\
& +e_{x}^{T}(t)\left(\widetilde{A}^{T} P+P \widetilde{A}\right) e_{x}(t)+\Psi^{T} P e_{x}(t)+e_{x}^{T}(t) P \Psi .
\end{aligned}
$$

Clearly, if

$$
e_{x}^{T}(t)\left(\widetilde{A}^{T} P+P \widetilde{A}\right) e_{x}(t)+\Psi^{T} P e_{x}(t)+e_{x}^{T}(t) P \Psi<0,
$$

then $\dot{V}(t)<0$, which implies that the fractional-order state estimation error (19) and the conventional integer-order fault estimation error (20) are asymptotically stable.

By using lemma 2 and equation (11), we obtain the following inequality

$e_{x}^{T}(t) P \Psi+\Psi^{T} P e_{x}(t) \leqslant \delta_{1}^{-1} e_{x}^{T}(t) P^{2} e_{x}(t)+\delta_{1} \lambda^{2} e_{x}^{T}(t) e_{x}(t)$

for any positive number $\delta_{1}$.

Substituting (27) into (26), we obtain the following sufficient condition

$$
\widetilde{A}^{T} P+P \widetilde{A}+\delta_{1}^{-1} P^{2}+\delta_{1} \lambda^{2} I<0,
$$

which is equivalent to

$$
(A-L C)^{T} P+P(A-L C)+\delta_{1}^{-1} P^{2}+\delta_{1} \lambda^{2} I<0 .
$$

By setting $P L=X$, we have

$$
P A+A^{T} P-X C-C^{T} X^{T}+\delta_{1}^{-1} P^{2}+\delta_{1} \lambda^{2} I<0 .
$$

Inequality (30) is equivalent to (16a) by the Schur complement [23]. This ends the proof.

Remark 2: In particular, simply setting $P=\beta I, X=\beta L$ and $Q=\beta A-X C$, the stability condition (16a) is equivalent to

$$
\left[\begin{array}{cc}
\frac{Q+Q^{T}}{2}+\frac{\delta_{1}}{2} \lambda^{2} I & \beta I \\
\bullet & -2 \delta_{1} I
\end{array}\right]<0
$$

Remark 3: The inequality (16) in theorem 1 can be solved by LMI toolbox, but solving difficulty is added because of the equation $G^{T} P=F C$. We can transform $G^{T} P=F C$ into the following LMI optimization problem [24], [25], [26],

$$
\text { Minimize } \rho \text { such that : }\left[\begin{array}{cc}
\rho I_{n} & G^{T} P-F C \\
\bullet & \rho I_{n}
\end{array}\right]>0
$$

where $\rho$ is a positive scalar. In order to make $G^{T} P$ approximate to $F C$ with satisfactory precision, a sufficiently small positive scalar $\rho$ should be selected in advance to meet (32).

Remark 4: From (17), the on-line fault estimator is as follows

$$
\widehat{f}(t)=-\Sigma F \int_{t_{f}}^{t} e_{y}(\tau) \mathrm{d} \tau
$$

where $t_{f}$ denotes the instant when fault occurs. 
This conventional integer-order (IO) adaptive fault estimator is only an integral term despite it can guarantee that the estimate of constant fault is unbiased, it fails to deal with time-varying faults. Therefore, we are motivated to improve the conventional IO adaptive fault estimation algorithm so that time-varying faults can be considered using FO adaptive observer, which is one of focuses in this next section.

\section{B. Fast fractional-order adaptive fault estimation}

Here, we consider time-varying faults, rather than constant faults. Due to $D^{\alpha} f(t) \neq 0$, the fractional-order derivative of $e_{f}(t)$ with respect to time is

$$
D^{\alpha} e_{f}(t)=D^{\alpha} \widehat{f}(t)-D^{\alpha} f(t)
$$

The asymptotical convergence of the fast fractional-order state estimation error and the fractional-order fault estimation error are given by the following theorem.

Theorem 2: If there exists matrices $P=P^{T}>0 \in$ $\mathbb{R}^{n \times n}, X \in \mathbb{R}^{m \times n}, F \in \mathbb{R}^{r \times p}$ and three real scalars $\delta_{1}>0, \delta_{2}>0$ and $\rho>0$ such that the following conditions hold

$$
\begin{gathered}
{\left[\begin{array}{cccc}
\Xi_{11} & 0 & \Xi_{13} & P \\
\bullet & -\delta_{2} I & -P G & 0 \\
\bullet & \bullet & \Xi_{33} & 0 \\
\bullet & \bullet & \bullet & -\delta_{1} I
\end{array}\right]<0} \\
{\left[\begin{array}{cc}
\rho I_{n} & G^{T} P-F C \\
\bullet & \multicolumn{2}{c}{\rho I_{n}}
\end{array}\right]>0}
\end{gathered}
$$

where

$$
\begin{aligned}
& \Xi_{11}=A^{T} P+P A-X C-C^{T} X^{T}+\left(\delta_{1}+\delta_{2}\right) \lambda^{2} I \\
& \Xi_{13}=-A^{T} P G+C^{T} X^{T} G \\
& \Xi_{33}=-G^{T} P G .
\end{aligned}
$$

Then the fractional-order adaptive fault estimation algorithm

$$
D^{\alpha} \widehat{f}(t)=-\Sigma F\left(D^{\alpha} e_{y}(t)+e_{y}(t)\right),
$$

can realize the state estimation error $e_{x}(t)$ and the fault estimation error $e_{f}(t)$ uniformly bounded where $\Sigma=\Sigma^{T}>$ $0 \in \mathbb{R}^{r \times r}$ is the learning rate.

Moreover, the asymptotically stabilizing state-feedback gain matrix $L$ is given by

$$
L=P^{-1} X \text {. }
$$

Proof: It follows from lemma 1 that the fractional-order observer error dynamic system (14) can be written as

$$
\left\{\begin{array}{l}
\frac{\partial Z_{1}(\omega, t)}{\partial t}=-\omega Z_{1}(\omega, t)+\widetilde{A} e_{x}(t)+G e_{f}(t)+\Psi \\
e_{x}(t)=\int_{0}^{\infty} \mu(\omega) Z_{1}(\omega, t) \mathrm{d} \omega
\end{array}\right.
$$

and the fractional-order fault estimation error (36) is equivalent to

$$
\left\{\begin{array}{l}
\frac{\partial Z_{2}(\omega, t)}{\partial t}=-\omega Z_{2}(\omega, t)-\Sigma F\left(D^{\alpha} e_{y}(t)+e_{y}(t)\right) \\
e_{f}(t)=\int_{0}^{\infty} \mu(\omega) Z_{2}(\omega, t) \mathrm{d} \omega
\end{array}\right.
$$

where $\widetilde{A}=A-L C$ and $\Psi=\Phi(x(t), u(t))-\Phi(\widehat{x}(t), u(t))$.

Let us define two Lyapunov functions

- $v(\omega, t)$ is the monochromatic Lyapunov function corresponding to the elementary frequency $\omega$.

- $V(t)$ is the Lyapunov function summing all the monochromatic $v(\omega, t)$ with the weighting function $\mu(\omega)$.

Thus, we can define our monochromatic Lyapunov function as

$$
\begin{aligned}
V(t)=\int_{0}^{\infty} \mu(\omega) & v(\omega, t) \mathrm{d} \omega=\int_{0}^{\infty} \mu(\omega) Z_{1}^{T}(\omega, t) P Z_{1}(\omega, t) \mathrm{d} \omega \\
& +\int_{0}^{\infty} \mu(\omega) Z_{2}^{T}(\omega, t) \Sigma^{-1} Z_{2}(\omega, t) \mathrm{d} \omega
\end{aligned}
$$

The time derivative of $V(t)$ taken along the solution trajectories of (38) is

$\dot{V}(t)=$

$\int_{0}^{\infty} \mu(\omega)\left(-\omega Z_{1}^{T}(\omega, t)+e_{x}^{T}(t) \widetilde{A}^{T}+e_{f}^{T}(t) G^{T}+\Psi^{T}\right) P Z_{1}(\omega, t) \mathrm{d} \omega$

$+\int_{0}^{\infty} \mu(\omega) Z_{1}^{T}(\omega, t) P\left(-\omega Z_{1}(\omega, t)+\widetilde{A} e_{x}(t)+G e_{f}(t)+\Psi\right) \mathrm{d} \omega$

$+\int_{0}^{\infty} \mu(\omega)\left(-\omega Z_{2}^{T}(\omega, t)-\left(\left(D^{\alpha} e_{y}\right)^{T}(t)+e_{y}^{T}(t)\right)\right) F^{T} \Sigma^{T}$

$\times \Sigma^{-1} Z_{2}(\omega, t) \mathrm{d} \omega$

$+\int_{0}^{\infty} \mu(\omega) Z_{2}^{T}(\omega, t) \Sigma^{-1}\left(-\omega Z_{2}(\omega, t)-\Sigma F\left(D^{\alpha} e_{y}(t)+e_{y}(t)\right)\right) \mathrm{d} \omega$.

Equation (41) can be written as

$$
\begin{aligned}
& \dot{V}(t)=-2 \int_{0}^{\infty} \omega \mu(\omega) Z_{1}^{T}(\omega, t) P Z_{1}(\omega, t) \mathrm{d} \omega \\
& \quad+\int_{0}^{\infty} \mu(\omega) e_{x}^{T}(t) \widetilde{A}^{T} P Z_{1}(\omega, t) \mathrm{d} \omega+\int_{0}^{\infty} \mu(\omega) Z_{1}^{T}(\omega, t) P \widetilde{A} e_{x}(t) \mathrm{d} \omega \\
& \quad+\int_{0}^{\infty} \mu(\omega) e_{f}^{T}(t) G^{T} P Z_{1}(\omega, t) \mathrm{d} \omega+\int_{0}^{\infty} \mu(\omega) Z_{1}^{T}(\omega, t) P G e_{f}(t) \mathrm{d} \omega \\
& \quad+\int_{0}^{\infty} \mu(\omega) \Psi^{T} P Z_{1}(\omega, t) \mathrm{d} \omega+\int_{0}^{\infty} \mu(\omega) Z_{1}^{T}(\omega, t) P \Psi \mathrm{d} \omega \\
& \quad-2 \int_{0}^{\infty} \omega \mu(\omega) Z_{2}^{T}(\omega, t) \Sigma^{-1} Z_{2}(\omega, t) \mathrm{d} \omega \\
& \quad-\int_{0}^{\infty} \mu(\omega)\left(\left(D^{\alpha} e_{y}\right)^{T}(t)+e_{y}^{T}(t)\right) F^{T} Z_{2}(\omega, t) \mathrm{d} \omega \\
& \quad-\int_{0}^{\infty} \mu(\omega) Z_{2}^{T}(\omega, t) F\left(D^{\alpha} e_{y}(t)+e_{y}(t)\right) \mathrm{d} \omega . \\
& =-2 \int_{0}^{\infty} \omega \mu(\omega) Z_{1}^{T}(\omega, t) P Z_{1}(\omega, t) \mathrm{d} \omega+e_{x}^{T}(t)\left(\widetilde{A}^{T} P+P \widetilde{A}\right) e_{x}(t) \\
& \quad-2 \int_{0}^{\infty} \omega \mu(\omega) Z_{2}^{T}(\omega, t) \Sigma^{-1} Z_{2}(\omega, t) \mathrm{d} \omega+2 e_{x}^{T}(t) P G e_{f}(t) \\
& -2 e_{f}^{T}(t) F C\left(D^{\alpha} e_{x}(t)+e_{x}(t)\right)+\Psi^{T} P e_{x}(t)+e_{x}^{T}(t) P \Psi . \quad(42)
\end{aligned}
$$

Using (16b), it is easy to show that

$$
\begin{aligned}
& -2 e_{f}^{T}(t) F C\left(D^{\alpha} e_{x}(t)+e_{x}(t)\right) \\
& \quad=-2 e_{f}^{T}(t) G^{T} P\left(D^{\alpha} e_{x}(t)+e_{x}(t)\right) .
\end{aligned}
$$

Substituting (43) into (42) yields

$$
\begin{aligned}
\dot{V}(t) & =-2 \int_{0}^{\infty} \omega \mu(\omega) Z_{1}^{T}(\omega, t) P Z_{1}(\omega, t) \mathrm{d} \omega \\
& -2 \int_{0}^{\infty} \omega \mu(\omega) Z_{2}^{T}(\omega, t) \Sigma^{-1} Z_{2}(\omega, t) \mathrm{d} \omega \\
+ & e_{x}^{T}(t)\left(\widetilde{A}^{T} P+P \widetilde{A}\right) e_{x}(t)-2 e_{f}^{T}(t) G^{T} P D^{\alpha} e_{x}(t)
\end{aligned}
$$




$$
+\Psi^{T} P e_{x}(t)+e_{x}^{T}(t) P \Psi
$$

By using lemma 2 and equation (11), we obtain the following inequality

$e_{x}^{T}(t) P \Psi+\Psi^{T} P e_{x}(t) \leqslant \delta_{1}^{-1} e_{x}^{T}(t) P^{2} e_{x}(t)+\delta_{1} \lambda^{2} e_{x}^{T}(t) e_{x}(t)$

for any positive number $\delta_{1}$.

Using (14) and substituting (45) into (44), we obtain the following inequality

$$
\begin{aligned}
\dot{V}(t) \leqslant & -2 \int_{0}^{\infty} \omega \mu(\omega) Z_{1}^{T}(\omega, t) P Z_{1}(\omega, t) \mathrm{d} \omega \\
& -2 \int_{0}^{\infty} \omega \mu(\omega) Z_{2}^{T}(\omega, t) \Sigma^{-1} Z_{2}(\omega, t) \mathrm{d} \omega \\
& +e_{x}^{T}(t)\left(\widetilde{A}^{T} P+P \widetilde{A}+\delta_{1}^{-1} P^{2}+\delta_{1} \lambda^{2} I\right) e_{x}(t) \\
& -2 e_{f}^{T}(t) G^{T} P \widetilde{A} e_{x}(t)-2 e_{f}^{T}(t) G^{T} P G e_{f}(t) \\
& -2 e_{f}^{T}(t) G^{T} P \Psi
\end{aligned}
$$

Since the nonlinear term $\Phi(x(t), u(t))$ satisfies the Lipschitz condition, so for a scalar $\delta_{2}>0$ we have

$$
\delta_{2} \lambda^{2} e_{x}^{T}(t) e_{x}(t)-\delta_{2} \Psi^{T} \Psi \geqslant 0
$$

From (46) and (47), one can further obtain that

$$
\begin{aligned}
\dot{V}(t) \leqslant & -2 \int_{0}^{\infty} \omega \mu(\omega) Z_{1}^{T}(\omega, t) P Z_{1}(\omega, t) \mathrm{d} \omega \\
& -2 \int_{0}^{\infty} \omega \mu(\omega) Z_{2}^{T}(\omega, t) \Sigma^{-1} Z_{2}(\omega, t) \mathrm{d} \omega \\
& +e_{x}^{T}(t)\left(\widetilde{A}^{T} P+P \widetilde{A}+\delta_{1}^{-1} P^{2}+\delta_{1} \lambda^{2} I\right) e_{x}(t) \\
& -2 e_{f}^{T}(t) G^{T} P \widetilde{A} e_{x}(t)-2 e_{f}^{T}(t) G^{T} P G e_{f}(t) \\
& -2 e_{f}^{T}(t) G^{T} P \Psi+\delta_{2} \lambda^{2} e_{x}^{T}(t) e_{x}(t)-\delta_{2} \Psi^{T} \Psi .
\end{aligned}
$$

By taking $X=P L$, we obtain

$$
\begin{aligned}
\dot{V}(t) \leqslant & -2 \int_{0}^{\infty} \omega \mu(\omega) Z_{1}^{T}(\omega, t) P Z_{1}(\omega, t) \mathrm{d} \omega \\
& -2 \int_{0}^{\infty} \omega \mu(\omega) Z_{2}^{T}(\omega, t) \Sigma^{-1} Z_{2}(\omega, t) \mathrm{d} \omega \\
& +e_{x}^{T}(t)\left(A^{T} P+P A-X C-C^{T} X^{T}\right. \\
& \left.+\delta_{1}^{-1} P^{2}+\left(\delta_{1}+\delta_{2}\right) \lambda^{2} I\right) e_{x}(t) \\
& -2 e_{f}^{T}(t)\left(G^{T} P A-G^{T} X C\right) e_{x}(t)-2 e_{f}^{T}(t) G^{T} P \Psi \\
& -2 e_{f}^{T}(t) G^{T} P G e_{f}(t)-\delta_{2} \Psi^{T} \Psi .
\end{aligned}
$$

Equation (49) can be written as

$$
\begin{aligned}
\dot{V}(t) \leqslant & -2 \int_{0}^{\infty} \omega \mu(\omega) Z_{1}^{T}(\omega, t) P Z_{1}(\omega, t) \mathrm{d} \omega \\
& -2 \int_{0}^{\infty} \omega \mu(\omega) Z_{2}^{T}(\omega, t) \Sigma^{-1} Z_{2}(\omega, t) \mathrm{d} \omega \\
& +\left[\begin{array}{c}
e_{x}(t) \\
\Psi \\
e_{f}(t)
\end{array}\right]^{T}\left[\begin{array}{ccc}
\Upsilon_{11} & 0 & \Upsilon_{13} \\
\bullet & -\delta_{2} I & -P G \\
\bullet & \bullet & \Upsilon_{33}
\end{array}\right]\left[\begin{array}{c}
e_{x}(t) \\
\Psi \\
e_{f}(t)
\end{array}\right]
\end{aligned}
$$

where

$$
\begin{aligned}
& \Upsilon_{11}=A^{T} P+P A-X C-C^{T} X^{T}+\delta_{1}^{-1} P^{2}+\left(\delta_{1}+\delta_{2}\right) \lambda^{2} I, \\
& \Upsilon_{13}=-A^{T} P G+C^{T} X^{T} G, \\
& \Upsilon_{33}=-G^{T} P G .
\end{aligned}
$$

Clearly, if

$$
\left[\begin{array}{ccc}
\Upsilon_{11} & 0 & \Upsilon_{13} \\
\bullet & -\delta_{2} I & -P G \\
\bullet & \bullet & \Upsilon_{33}
\end{array}\right]<0,
$$

then $\dot{V}(t)<0$, which implies that the fractional-order state estimation error (38) and the fractional-order fault estimation error (39) are asymptotically stable. Inequality (51) is equivalent to (35a) by the Schur complement [23]. This ends the proof.

Remark 5: From (36), the on-line fractional-order fault estimator is as follows

$$
\widehat{f}(t)=-\Sigma F\left(e_{y}(t)+\frac{1}{\Gamma(\alpha)} \int_{t_{f}}^{t}(t-\tau)^{\alpha-1} e_{y}(\tau) \mathrm{d} \tau\right)
$$

where $t_{f}$ denotes the instant when fault occurs.

The proposed fast fractional-order adaptive fault estimation algorithm combines proportional term with integral one. The introduction of the proportional term plays a major role to improve the rapidity an accuracy of the fault estimation.

\section{NUMERICAL EXAMPLE}

Consider the fractional-order nonlinear systems (10) with the fractional-order $\alpha=0.9$ and the following parameters

$$
\left\{\begin{array}{l}
A=\left[\begin{array}{cc}
1 & 1 \\
-1 & 1
\end{array}\right], \quad \Phi(x)=\left[\begin{array}{c}
0 \\
0.05 \sin \left(x_{2}\right)
\end{array}\right], \\
C=\left[\begin{array}{ll}
1 & 0
\end{array}\right], \quad G=\left[\begin{array}{l}
1 \\
1
\end{array}\right] .
\end{array}\right.
$$

It can be easily shown that the pair $(C, A)$ is observable and $\operatorname{rank}(C G)=1$. Choosing $\lambda=0.05$ and $\rho=1.2 \times 10^{-2}$, then the solution of the LMIs (35a) and (35b) with respect to $P, X$ and $F$ gives

$$
\begin{aligned}
& P=\left[\begin{array}{cc}
0.2577 & -0.1405 \\
-0.1405 & 0.0835
\end{array}\right], \quad X=\left[\begin{array}{c}
0.5712 \\
-0.2879
\end{array}\right], \\
& F=[0.1173], \quad \delta_{1}=1.0467, \quad \delta_{2}=1.512,
\end{aligned}
$$

and the gain matrix $L$ is given by

$$
L=X P^{-1}=\left[\begin{array}{l}
4.0560 \\
3.3741
\end{array}\right] \text {. }
$$

We illustrate the results with time-varying faults which is created as

$$
f(t)= \begin{cases}0 & 0 \leqslant t \leqslant 2 \\ 0.4 \sin (2 t) & 2 \leqslant t \leqslant 10 .\end{cases}
$$

The trajectories of the states and their estimates are shown in Figs. 1 and 2. It can be seen that the proposed fast fractional-order adaptive observer is asymptotically stable and its states variables appear to be well estimated.

From Fig. 3, we can see that the fast fractional-order adaptive fault estimator can achieve asymptotical estimation for the time varying-fault. 


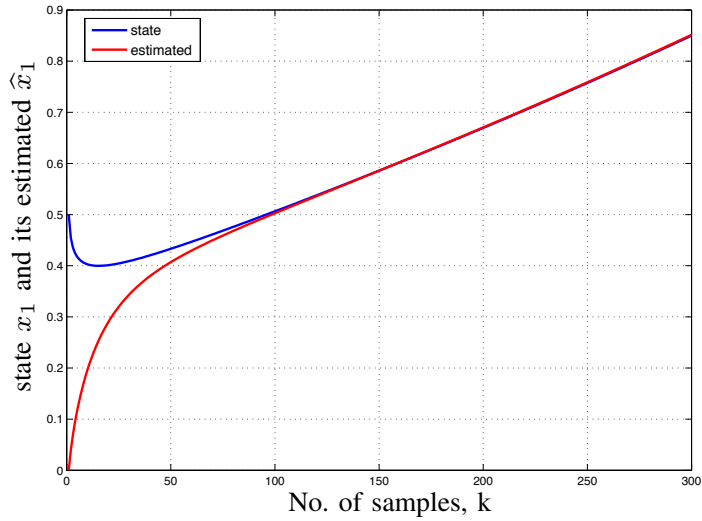

Fig. 1. State $x_{1}$ and its estimated $\widehat{x}_{1}$ where $\alpha=0.9$.

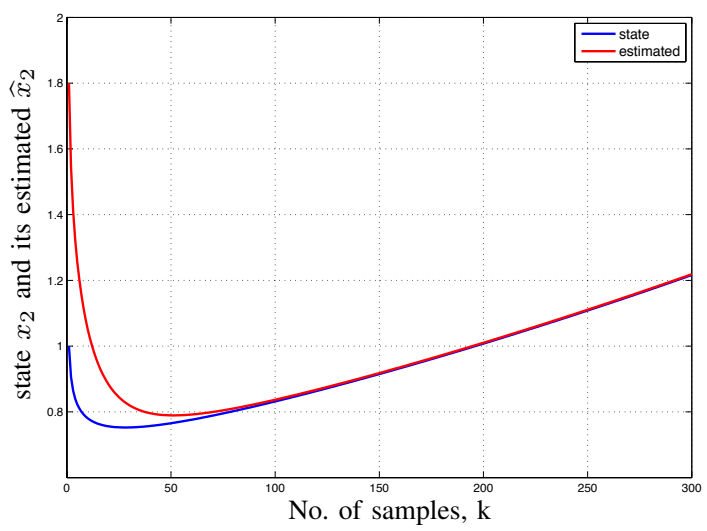

Fig. 2. State $x_{2}$ and its estimated $\widehat{x}_{2}$ where $\alpha=0.9$.

\section{CONCLUSION}

In this paper, the fractional-order fault estimation observer design for a class of nonlinear fractional-order systems has been presented. Based on the continuous frequency distributed equivalent model and indirect Lyapunov approach, sufficient conditions for the asymptotical convergence of the fractional-order state estimation error, the conventional integer-order and the fractional-order faults estimation error have been derived in terms of linear matrix inequalities (LMIs) formulation where the fractional-order $\alpha$ belongs to $0<\alpha<1$. A numerical example has been illustrated to demonstrate the effectiveness of the proposed approach.

\section{ACKNOWLEDGEMENT}

The research reported herein is supported by the King Abdullah University of Science and Technology (KAUST).

\section{REFERENCES}

[1] N. Engheta, "On fractional calculus and fractional multipoles in electromagnetism," IEEE Trans. Antennas and Propagation, vol. 44, pp. 554-566, 1996.

[2] H. Sun, A. Abdelwahad, and B. Onaral, "Linear approximation of transfer function with a pole of fractional order," IEEE Trans. Aut. Contr, vol. 29, pp. 441-444, 1984.

[3] R. Bagley and R. Calico, "Fractional order state equations for the control of viscoelastically damped structures," J. Guidance, Contr. \& Dynamics, vol. 14, pp. 304-311, 1991.

[4] K. Cole, "Electric conductance of biological systems," in Proc. Cold Spring Harbor Symp. Quantitative Biology, (New York, USA), pp. 107-116, 1933.

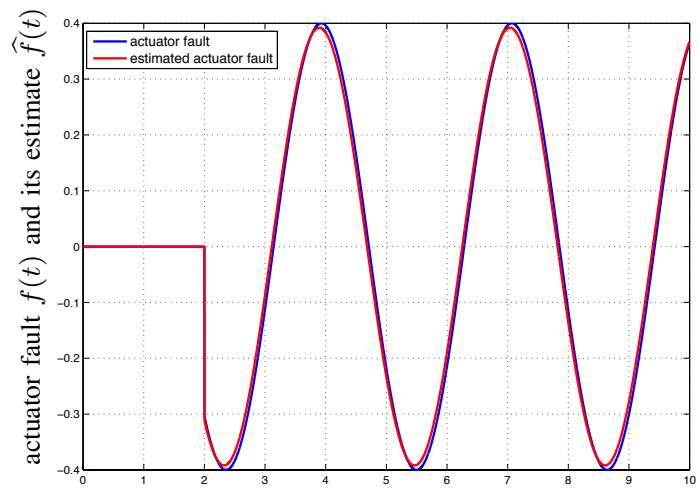

Fig. 3. Actuator fault $f(t)$ and its estimate $\widehat{f}(t)$ with $\alpha=0.9$.

[5] N. Laskin, "Fractional market dynamics," Physica A: Statistical Mechanics and its Applications, vol. 287, pp. 482-492, 2000.

[6] J. Trigeassou, N. Maamri, J. Sabatier, and A. Oustaloup, "A Lyapunov approach to the stability of fractional differential equations," Signal Processing, vol. 91, pp. 437-445, 2011.

[7] J. Tsinias, "Observer design for nonlinear systems," Syst. \& Contr. Letters, vol. 13, pp. 135-142, 1989.

[8] J. Gauthier, H. Hammouri, and S. Othman, "A simple observer for nonlinear systems, applications to bioreactors," IEEE Trans. Aut. Contr., vol. 37, pp. 875-880, 1992.

[9] S. Raghavan and J. Hedrick, "Observer design for a class of nonlinear systems," Int. J. Contr., vol. 59, pp. 515-528, 1994.

[10] R. Rajamani, "Observer for Lipschitz nonlinear systems," IEEE Trans. Aut. Contr., vol. 43, pp. 397-401, 1998.

[11] E. Sontag and Y. Wang, "Output-to-state stability and detectability of nonlinear systems," Syst. \& Contr. Letters, vol. 29, pp. 279-290, 1997.

[12] J. Hespanha and A. Morse, "Certainty equivalence implies detectability," Syst. \& Contr. Letters, vol. 36, pp. 1-13, 1999.

[13] E. A. Boroujeni and H. R. Momeni, "Non-fragile nonlinear fractional order observer design for a class of nonlinear fractional order systems," Signal Processing, vol. 92, pp. 2365-2370, 2012.

[14] Y. H. Lang and Y. Zhou, "Non-fragile observer-based robust control for a class of fractional-order nonlinear systems," Syst. \& Contr. Letters, vol. 62, pp. 1143-1150, 2013.

[15] H. Wang and S. Daley, "Actuator fault diagnosis: An adaptive observer-based technique," IEEE Trans. Aut. Contr., vol. 41, pp. 1073 1078, 1996

[16] M. R. Rapaić and A. Pisano, "Variable-order fractional operators for adaptive order and parameter estimation," IEEE Trans. Aut. Contr. vol. 59, pp. 798-803, 2014.

[17] D. Y. Liu and T. M. Laleg-Kirati, "Robust fractional order differentiators using generalized modulating functions method," Signal Processing, vol. 107, pp. 395-406, 2014.

[18] S. Das, Functional Fractional Calculus for System Identification and Controls. Heidelberg: Springer, 2008.

[19] I. Podlubny, Fractional Differential Equations. New York: Academic Press, 1999.

[20] P. Khargonakar, I. Petersen, and K. Zhou, "Robust stabilization of uncertain linear systems : quadratic stability and $\mathcal{H}_{\infty}$ control theory," IEEE Trans. Aut. Contr., vol. 35, pp. 356-361, 1990.

[21] D. Matignon and B. Andréa-Novel, "Observer-based for fractional differential systems," in Proc. IEEE Conf. Decision \& Contr., (San Diego, USA), 1997.

[22] M. Darouach, M. Zasadzinski, and S. Xu, "Full-order observers for linear systems with unknown inputs," IEEE Trans. Aut. Contr., vol. 39 , pp. 606-609, 1994.

[23] S. Boyd, L. El Ghaoui, E. Féron, and V. Balakrishnan, Linear Matrix Inequality in Systems and Control Theory. Philadelphia: SIAM, 1994.

[24] M. Corless and J. Tu, "State and input estimation for a class of uncertain systems," Automatica, vol. 34, pp. 757-764, 1998.

[25] K. Zhang, B. Jiang, and V. Cocquempot, "Adaptive observer-based fast fault estimation," Int. J. Cont., Autom. Syst., vol. 6, pp. 320-326, 2008

[26] K. Zhang, B. Jiang, and P. Shi, Observer-Based Fault Estimation and Accommodation for Dynamic Systems. Berlin: Springer-Verlag, 2nd ed., 2013. 